$\Delta_{\text {Vol 1, No. } 3 \text { Sep / Oct } 2016}$

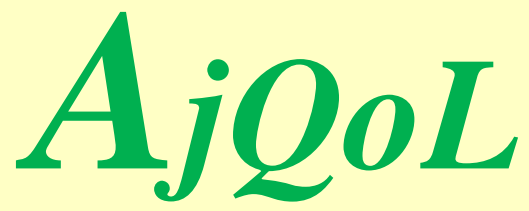

Asian Journal of Quality of Life

elSSN: 2398-4279

\title{
Conscientiousness and Collectivism as the Predictors of Shame and Guilt Proneness
} Juneman Abraham, Murty Magda Pane

\section{Efficacy and Decisional Balance to Intervene}

Tommy Prayoga, Amanda Giovani Pea, Yosef Dedy Pradipto

Integration of Supply Chain in IBS Construction

Ani Saifuza Abd Shukor, Faridah Muhammad Halil, Mohammad Fadhil Mohammad, Rohana Mahbub

\author{
Metaphysics Relevancy in Contemporary Design \\ Masran Saruwono, Nor Aniswati Awang Lah
}

Vertical Greening Wall As Sustainable Approach
Ahmad Ridzwan Othman, Norshamira Sahidin

A bi-monthly international publication by e-IPH, Ltd., UK, for the 
Published by:

International Publishing House (e-IPH), Limited

(Registration No: 10062603, for England \& Wales)

18 Westpoint 58 West Street

Sheffield S1 4EZ

United Kingdom 


\section{Asian Journal}

Vol.1, No. 03, Sep / Oct 2016
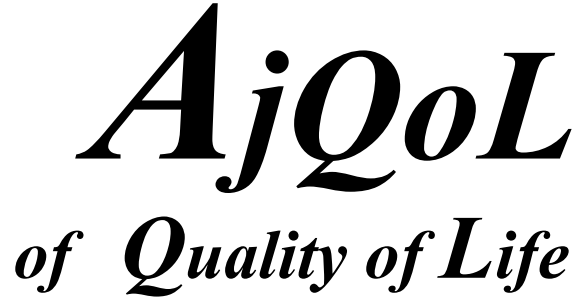

elSSN 2398-4279

Conscientiousness and Collectivism as the Predictors of Shame and Guilt Proneness Juneman Abraham, Murty Magda Pane

Efficacy and Decisional Balance to Intervene

Tommy Prayoga, Amanda Giovani Pea, Yosef Dedy Pradipto

Integration of Supply Chain in IBS Construction

Ani Saifuza Abd Shukor, Faridah Muhammad Halil, Mohammad Fadhil Mohammad, Rohana Mahbub

Metaphysics Relevancy in Contemporary Design $29-38$ Masran Saruwono, Nor Aniswati Awang Lah

Vertical Greening Wall As Sustainable Approach

39-51

Ahmad Ridzwan Othman, Norshamira Sahidin 
Asian Journal of Quality of Life, AjQoL,1(3), Sep / Oct 2016 


\section{Foreword}

The Asian Journal of Quality of Life (AjQoL), is an international and interdisciplinary Open Access Journal. It publicises online both conceptual and empirical research findings pertaining to quality of life issues affecting Asian communities living in Asia and non-Asian countries. Related studies that involve other communities shall also be considered for publications.

Articles published are sourced from condensed and retitled, among the best full papers, internationally double-blind, peer-reviewed presented at serial international conferences held worldwide since 2010, namely, the AicQoL (AMER International Conference on Quality of Life) held in ASEAN countries,, and AQoL (ABRA International Conference on Quality of Life) held in non-ASEAN countries (except Malaysia). There are no processing and publication fees for those successful articles to be published.

Those conferences are jointly organised by AMER (Association of Malaysian Environment-Behaviour Researchers); ABRA (Assocation of Behavioural Researchers on Asians); and the $c E-B s$ (Centre for Environment-Behaviour Studies), Faculty of Architecture, Planning \& Surveying, Universiti Teknologi MARA, Malaysia. International institutions co-hosted the conferences.

Currently, the Editorial Board (Advisory/Reviewer) Members comprises of 69 academicians represented from 17 countries worldwide, namely, Australia, Bahrain, Cyprus, Egypt, Hong Kong, India, Indonesia, Iran, Japan, Malaysia, Nigeria, North Cyprus, Taiwan, Thailand, Turkey, U.K, and U.S.A.

The AjQoL shall be published online bimonthly, and freely accessible at the web link below. All articles published are with the Doi and Crossmark Reference.

\section{Editor-in-Chief}

Asian Journal of Quality of Life (AjQoL), elSSN 2398-4279

http://ajgol.e-iph.co.uk

myusoff801@salam.uitm.edu.my

01 Sep 2016 
Asian Journal of Quality of Life, AjQoL,1(3), Sep / Oct 2016 


\section{Editorial Team Members}

\section{Editor-in-Chief}

Mohamed Yusoff Abbas, Prof., Dr.

myusoff801@salam.uitm.edu.my

\section{Associate Editors}

\section{Egypt}

Rasha Mahmoud Ali El-Zeiny, Assoc Prof.Dr. Menia University, Menia, Egypt

rasha_elzeiny@yahoo.com

India

Smita Khan, Assoc. Prof., Dr., Ar.

smitakhan@gmail.com

Indonesia
Faculty of Architecture, Planning \& Surveying Universiti Teknologi MARA

40450 Shah Alam, Malaysia
Juneman Abraham

juneman@binusian.org

Iran

Mona Bisadi, Dr.

bisadi.mona@gmail.com

Taiwan

Shenglin Elijah Chang, Prof., Dr.

shenglin@ntu.edu.tw

Turkey

Ebru Cubukcu, Prof., Dr.

Dokuz Eylul University, Turkey

ebru.cubukcu@deu.edu.tr

Bina Nusantara University, Jakarta, Indonesia

Islamic Azad University, Tehran, Iran

National Taiwan University, Taiwan

\section{Editorial Board (Advisory/Reviewer) Members}

Abdul Hadi Nawawi, Prof., Dr

FAPS, Universiti Teknologi MARA, Malaysia

abdul274@salam.uitm.edu.my

Aldrin Abdullah, Prof., Dr.

Universiti Sains Malaysia, Malaysia

aldrin@usm.my 
Aleya Abdel Hadi, Emiretus Prof., Dr.

aabdelhadi@msa.eun.eg

Anuar Talib, Assoc. Prof., Dr.

anuarfaqir@gmail.com

Ashraf M. Salama, Prof. Dr.

ashraf.salama@strath.ac.uk

Babatunde Emmanuel Jaiyeoba, Dr.

tundejaiyeoba@yahoo.co.uk

Christopher Peter Spencer, Emer. Prof., Dr. C.P.Spencer@sheffield.ac.uk

Cigdem Canbay Turkyilmaz, Assoc. Prof., Dr. cigdemcanbay@gmail.com

Darussalam Abu Bakar, Prof., Dr.

darussal@salam.uitm.edu.my

Dasimah Omar, Prof., Dr.

dasimah629@salam.uitm.edu.my

David Dernie, Prof., Dr.

D.Dernie@westminster.ac.uk

David Robert Osborne, Dr.

dro@graphiabrands.com

Edward Andrew Edgerton, Dr.

Edward.Edgerton@uws.ac.uk

Elizabeth Grant, Dr.

elizabeth.grant@adelaide.edu.au

Emrah Turkyilmaz, Dr.

e.turkyilmaz@iku.edu.tr

Faridah Ismail, Assoc. Prof., Dr.

farid346@salam.uitm.edu.my

Filiz Çelik, Asst. Prof., Dr.

filiz@selcuk.edu.tr
FAD, Modern Sciences \& Arts University, Egypt

FAPS, Universiti Teknologi MARA, Malaysia

University of Strathclyde, Glasgow, UK

ObafemiAwolowo University, lle-lfe, Nigeria

The University of Sheffield, UK

Yildiz Technical University, Turkey

FKOM, Universiti Teknologi MARA, Malaysia

FAPS, Universiti Teknologi MARA, Malaysia

University of Westminster, UK

Graphia International Limited, Hong Kong

West of Scotland University, UK

The University of Adelaide, Australia

Istanbul Kultur University, Turkey

FAPS, Universiti Teknologi MARA, Malaysia

Selcuk University, Konya-Turkey 
Hazlina Hamdan, Assoc. Prof., Dr.

hazlina594@salam.uitm.edu.my

Hazreena Hussein, Dr.

reenalambina@um.edu.my

Himasari Hanan, Dr., Ir.

hanan@ar.itb.ac.id

Heng Zhang, Prof., Dr., Ing.

changlin@mail.ncku.edu.tw

Ismail Said, Assoc. Prof., Dr.

b-ismail@utm.my

Ismail Samsuddin, Assoc. Prof., Dr.

ismai587@perak.uitm.edu.my

Iwan Sudradjat, Prof., Dr.

iwansudr@ar.itb.ac.id

Jamalunlaili Abdullah, Assoc. Prof., Dr.

jamal858@salam.uitm.edu.my

Jin-Yung Wu, Asst. Prof., Dr., Ar.

jinyungwu@gmail.com

Lobna Abdel Aziz Ahmed, Assoc. Prof., Dr.

Arch.Lobna@gmail.com

Mariam Jamaluddin, Assoc. Prof., Dr., Ar. marja67@gmail.com

Masahiro Shirotsuki, Dr.

mshiro@nufs.ac.jp

Masran Saruwono, Assoc. Prof., Dr.

masran697@salam.uitm.edu.my

Mazlina Mansor, Asst. Prof., Dr. mazlina.mansor@gmail.com

Mei Lim, Dr.

Mei.Lim@unisa.edu.au
FAPS, Universiti Teknologi MARA, Malaysia

FBE, University Malaya, Malaysia

Institut Teknologi Bandung, Indonesia

National Cheng Kung University, Taiwan

FBE, Universiti Teknologi Malaysia, Malaysia

FAPS, Universiti Teknologi MARA Perak, Malaysia

Institut Teknologi Bandung, Indonesia

FAPS, Universiti Teknologi MARA, Malaysia

DAS, National Taiwan University, Taipei, Taiwan

Arch. Dept., Menia University, Menia, Egypt

FAPS, Universiti Teknologi MARA, Malaysia

SCIS, Nagoya University, Japan

FAPS, Universiti Teknologi MARA, Malaysia

KAED, International Islamic University Malaysia

University of South Australia, Australia 
Mert Cubukcu, Prof., Dr.

mert.cubukcu@deu.edu.tr

Mohammad Arif Kamal, Assoc. Prof, Dr.

architectarif@gmail.com

Mohd Sabrizaa Abd Rashid, Assoc. Prof., Dr mohds637@perak.uitm.edu.my

Nicholas Kathijotes, Prof., Dr.

nicholas.kathijotes@cut.ac.cy

Nisha Fernando, Prof., Dr., Ar.

Nisha.Fernando@uwsp.edu

Nor Haslina Jaafar, Dr.

mellina.inamel@gmail.com

Nor Zalina Harun, Dr.

nzalina76@gmail.com

Noor Hanita Abdul Majid, Asst. Prof., Dr.

hanita@iium.edu.my

Norhati Ibrahim, Assoc. Prof., Dr., Ar.

norhati@salam.uitm.edu.my

Noriah Othman, Dr.

noriaho@yahoo.com

Norsidah Ujang, Assoc. Prof., Dr.

norsidah@putra.upm.edu.my

Nurlisa Ginting, Assoc. Prof., Dr.

nurlisa.ginting@gmail.com

Osman Mohd Tahir, Assoc. Prof., Dr.

osman mtahir@yahoo.com

Paramita Atmodiwirjo, Assoc. Prof., Dr.

$\underline{\text { mitayandi@gmail.com }}$

Prabu Wardono, Dr.

pwardono@yahoo.com
Dokuz Eylul University, Turkey

Arch. Sec., Aligarh Muslim University, India

FAPS, Universiti Teknologi MARA Perak, Malaysia

Cyprus University of Technology, Cyprus

University of Wisconsin, USA

Universiti Kebangsaan Malaysia, Malaysia

KAED, International Islamic University Malaysia

KAED, International Islamic University Malaysia

FAPS, Universiti Teknologi MARA, Malaysia

FAPS, Universiti Teknologi MARA, Malaysia

Universiti Putra Malaysia, Malaysia

Universiti Sumatera Utara, Medan, Indonesia

Universiti Putra Malaysia, Malaysia

Universitas Indonesia, Indonesia

Institut Teknologi Bandung, Indonesia 
Ranjith Dayaratne, Assoc. Prof., Dr.

ranjith.dayaratne@gmail.com

Robert W. Marans, Emer. Prof., Dr.

marans@umich.edu

Rugayah Gy Hashim, Assoc. Prof., Dr. gy@salam.uitm.edu.my

Sabarinah Sh. Ahmad, Prof., Dr.

sabar643@salam.uitm.edu.my

Saripah Abdul Latif

saripah@kelantan.uitm.edu.my

Sertaç Gungor, Asst. Prof., Dr.

sertac@selcuk.edu.tr

Shahab Kariminia, Asst. Prof., Dr.

sh.kariminia@yahoo.com

Siriwan Silapacharanan, Prof., Dr.

siriwan.s@chula.ac.th

Sitthiporn Piromruen, Assoc. Prof.

sitthip128@yahoo.com

Siti Rukayah, Dr., Ir.

tututrsiti@yahoo.com

Supagtra Suthasupa, Asst. Prof., Dr.

supakoy@yahoo.com

Uday Jain, Prof., Dr.

jainuday1941@gmail.com

Wan Hashimah Wan Ismail, Dr.

wan7137shima@gmail.com

Wannasilpa Peerapun, Prof., Dr.

Wannasilpa.P@chula.ac.th

Zehra Öngül, Asst. Prof.

zehra.ongul@emu.edu.tr
University of Bahrain, Bahrain

University of Michigan, Michigan, USA

FSPPP, Universiti Teknologi MARA, Malaysia

FAPS, Universiti Teknologi MARA, Malaysia

Universiti Teknologi MARA, Kelantan, Malaysia

Selcuk University, Konya, Turkey

Islamic Azad University, Isfahan, Iran

Chulalongkorn University, Thailand

Silpakorn University, Thailand

Diponegoro University, Semarang, Indonesia

Silpakorn University, Thailand

Mahila Chetna Manch, Shivaji Nagar, India

FBE, Universiti Teknologi Malaysia, Malaysia

Chulalongkorn University, Bangkok, Thailand

Eastern Mediterranean University, North Cyprus 


\section{Assistant Editor-In-Chief}

Syazwani Abdul Kadir

\section{Editorial Assistants}

Diyanah Inani Azmi

Maimunah Ramlee

Mohd Fauzee Musa

Mohd Riduan Ngesan

Sabrina Idilfitri

Sharifah Khalizah Syed Othman Thani

Siti Nur Afiqah Mohamed Musthafa

Suhaini Mohamed Yusoff 